\title{
Current Concepts on Immune Regulation: Impact on Scientific and Clinical Aspects of Immunohematology
}

\author{
Dorothea Stahl \\ Institut für Transfusionsmedizin, Westfälische Wilhelms-Universität Münster, Germany
}

Immunohematology aims at predicting by in vitro assays the in vivo compatibility of blood, cells, and tissues in the setting of transfusion and transplantation medicine. Immunohematology therefore focuses on aspects of blood cell antigen typing, and on the detection and characterization of humoral and cellular immune responses to blood cell antigens. Progress in immunohematology in the past has been defined mainly by the application of state-of-the-art techniques in order to enhance the level of certitude with which to predict the degree of in vivo compatibility. Thus, serological techniques to type blood cell antigens have been supplemented by approaches derived from the methodological spectrum of molecular biology, and high throughput screening techniques such as cDNA- or oligonucleotide-based microarray formats are likely to complete the spectrum of immunohematological techniques during the coming years.

The concept of compatibility is dependent on the specificity of immune responses. According to the current understanding widely present within the immunohematology community, specificity is defined by structural components of single cells, single antibodies, and their interaction. However, beyondgenome biology indicates strongly that the specificity of functional modules of the immune system contributes to the overall outcome of immune responses. Thus, specificity - and thereby compatibility - may be defined by functional aspects of the immune system in its respective condition as much as by structural components of immune cells and immune receptors. Such an altered concept of compatibility might have far reaching consequences for our understanding of tolerance to blood cell antigens with regard to autoimmune responses as well as with regard to alloimmune responses. It requires the understanding of regulatory mechanisms that determine the proper recognition of self and non-self antigens in the context of complex dynamic interactions at the cellular and humoral level of the immune system. Current concepts on immune regulation start to take into account the inherent complexity of biological systems. Future directions of immunohematological research might shift the focus of efforts from applying advances of compatibility testing at an exclusively structural level towards a functional understanding of compatibility, taking into account current concepts on immune regulation. The present issue and the follow-up issue of TRANSFUSION MEDICINE AND Hemotherapy present a series of reviews highlighting the current understanding of immune interactions that contribute to the outcome of immune responses, and that may be of relevance for the functional understanding of the phenomenon 'compatibility' in immunohematology.

In the first review, Arthur Silverstein, USA, introduces the topic of this issue by providing an overview on the development of concepts on the understanding of the immune response in the historical context since the end of the 19th century, and demonstrates how the clarification of cellular and molecular regulatory mechanisms became one of the central interests of current immunobiology [1].

The current knowledge in immunobiology of diverse biological components and the data that are emerging from highthroughput screening technologies call for the need to understand how the individual genes, proteins, signaling pathways and molecular networks are integrated and how they govern the behavior of cells, tissues and physiological systems, allowing to dynamically maintain the organism's homeostasis. Systems analysis is regarded as an emerging interdisciplinary field of systems biology which relies upon the extensive use of a range of mathematical modeling tools, allowing the integration of various regulation levels. Gennady Bocharov, Russia, presents the methodology of systems analysis, and comprehensively reviews the application of systems analysis to complex regulatory phenomena in biology [2].

Jacques F.A.P. Miller, Australia, summarizes basic principles of immunological tolerance, depicting $\mathrm{T}$ lymphocytes and $\mathrm{B}$

\begin{tabular}{ll}
\hline KARGER & @ 2005 S. Karger GmbH, Freiburg \\
Fax +49 76145207 14 & Accessible online at: \\
$\begin{array}{l}\text { E-mail Information@Karger.de } \\
\text { www.karger.com }\end{array}$ & www.karger.com/tmh
\end{tabular}

PD Dr. med. Dorothea Stahl

Institut für Transfusionsmedizin

Westfälische Wilhelms-Universität Münster

Domagkstraße 11, 48149 Münster, Germany

Tel. +49 251 835-7312, Fax -8505

E-mailstahld@uni-muenster.de 
lymphocytes as key players in the setting of immune responses. Miller demonstrates that immunological tolerance is a multistep process, induced by a great variety of mechanisms influencing both $\mathrm{T}$ and $\mathrm{B}$ cells and their precursors [3].

The contribution of B cells to the outcome of immune responses essentially depends on the specificity of the B-cell receptor (BCR). Claudia Berek, Germany, reviews somatic hypermutation and BCR selection as regulators of the immune response: during B-cell development from the hematopoietic stem cell compartment, a primary repertoire of roughly $10^{10}$ different BCRs can be generated from just a few gene elements. Combinatorial diversity and junctional diversity provide the means of producing this large antibody repertoire with a broad spectrum of antigen specificities. However, the affinity of the BCR may be too low to support an efficient immune response to a given antigen. The B-cell immunoglobulin genes undergo additional refinements which allow the immune response to mature. As a result, the affinity of the BCR increases during the immune response, a process which is referred to as affinity maturation. Antigen-dependent activation of the immune system will induce the formation of germinal centers, a microenvironment where the process of affinity maturation takes place through the interplay of somatic hypermutation and selection. Here, long lived memory and plasma cells are generated from a single antigen-activated B cell, and this ensures the continued protection of the organism with high-affinity antibodies. Antibody responses both to non-self and to self antigens originate from the selection and differentiation of antigen-activated B cells in germinal centers. Somatic mutation and selection serve to regulate the immune responses by fine tuning the affinity of the antibodies [4].

Tight regulation of B-cell activity is required to ensure humoral immunity against pathogens on the one hand and to avoid detrimental autoreactivity on the other. Activation of $\mathrm{B}$ cells is mediated through cross-binding of the BCR and is greatly enhanced by co-engagement of complement receptor type 2 (CD21, CR2) and its signaling element CD19. Feedback inhibition of B-cell activity is mediated by the binding of IgG antibodies to FcyRIIB (CD32). The extent to which the individual receptors are engaged depends on the context in which antigen is encountered by the B cells, be it as antigen alone, as complement-opsonized antigen, or as antigen complexed with IgM or IgG antibodies. Both overexpression of CD19 and deficiency of elements centrally involved in negative signaling, e.g. Fc $\gamma$ RIIB and Lyn, lead to autoimmune disease in mice. Upon formation of complement-activating immune complexes with natural or disease-associated antibodies, self antigen binds to B cells via CD21 and CD35 (CR1) and induces proliferation of self-reactive B cells and CD4+ T cells. Claus Henrik Nielsen and Robert Graham Quinton Leslie, Denmark, review the role of BCR, CD21/CD19 and Fc $\gamma$ RIIB in regulating $\mathrm{B}$-cell activity in immune and autoimmune responses [5].

Birgitta Heyman, Sweden, concentrates on the phenomenon of antibody feedback inhibition as a biological principle of immune regulation: antibodies passively administered together with their specific antigen are known to influence the antibody response to this antigen. Antibody feedback regulation can either enhance or suppress antibody responses. The effects are dramatic, frequently leading to $>99 \%$ suppression of the immune response or to enhancement of the immune response by $>1,000$-fold. IgG suppresses responses to large particulate antigens like erythrocytes and is used clinically to prevent $\mathrm{Rh}(\mathrm{D})$-negative women from becoming immunized against $\mathrm{Rh}(\mathrm{D})$-positive fetal erythrocytes during pregnancy. In experimental systems, this type of suppression works equally well in mice lacking the known Fc receptors for IgG and in wild-type animals. The mechanism is not known, but epitope masking by IgG would explain most of the experimental findings. Another type of IgG-mediated negative feedback regulation is exerted via the inhibitory Fc $\gamma$ RIIB. This inhibitory receptor negatively regulates - but does not completely prevent - the response to $\mathrm{IgG} /$ soluble protein antigens [6].

Aspects of immune complex processing are in the focus of the review of Mariano Sánchez Crespo, Spain. Antigen-antibody complex formation is a constant event during homeostatic inflammation, but it is currently overlooked as it is followed by an efficient removal of the complexes by phagocytosis under physiological conditions. However, immune complex-mediated tissue damage may occur under certain conditions. Current views about the mechanisms underlying immune complex-mediated tissue injury have emphasized the modulatory role on the outcome of inflammatory responses of the cooperation between antibody-driven phagocytosis and the recognition of pattern-associated molecular patterns (PAMP) which are expressed by microorganisms, by pattern recognition receptors (PRR) which are expressed by the host cells. Sánchez Crespo describes immune complex processing as a phagocytosis-based mechanism with proinflammatory potential, and highlights in particular the importance of signals conveyed via Fc $\gamma \mathrm{R}$ and PRR on the production of lipid mediators and chemokines in the context of immune complex-mediated tissue injury, and the cooperation between the Fc $\gamma \mathrm{R}$ route and the complement system in the safe removal of immune complexes [7].

Scientific knowledge on cells involved in and mechanisms contributing to antigen presentation have significantly expanded during the recent years. Dendritic cells are considered the most potent antigen-presenting cells because of their unique ability to initiate immune responses against invading pathogens. Dendritic cells are specialized in antigen transport to secondary lymphoid organs and subsequent stimulation of primary and secondary T-cell responses. Peptides presented by dendritic cells may also include peptides derived from self proteins, a process that may lead to the development of autoimmune disease. Elke Scandella and Burkhard Ludewig, Switzerland, provide an overview on current data on autoimmune reactivity mediated by dendritic cells, and on regulatory mechanisms limiting immunopathological damage [8]. 
Accumulating evidence indicates that B lymphocytes exert regulatory effects on the antigen-presenting function of dendritic cells. Srini V. Kaveri and colleagues, France, have recently shown that natural antibodies sustain the differentiation and maturation of human dendritic cells, and focus in their review on the effects of therapeutic intravenous immunoglobulins on the development and function of dendritic cells [9]. Interactions at the dendritic cell / T-cell interface that define the balance between immunity and tolerance are in the center of a review contributed by Mirentxu Iruretagoyena, Juan Lezana and Alexis Kalergis, Chile: considering that dendritic cells are the antigen-presenting cells determining the primary activation of naïve T cells, they play a pivotal role in the regulation of adaptive immunity and the maintenance of immune tolerance to self antigens. It is thought that $\mathrm{T}$ cells recognizing self constituents in the periphery are kept under control by immature dendritic cells through the presentation of self antigens in the absence of co-stimulation. Thus, alterations in the physiology of dendritic cells could be responsible for incomplete tolerance to self. Kalergis and colleagues concentrate in particular on the capacity of the immunological synapse occurring at the dendritic cell / T-cell interface to fine-tune the balance between tolerance and immunity. They describe how alterations of some of the constituents that participate in the synapse can determine induction or perpetuation of undesired immune responses. Physiological factors and pharmacological approaches modulating dendritic cell function and thus influencing the course of the immune response are also discussed [10].

Jan Buer and colleagues, Germany, present an update on mechanisms of central and peripheral T-cell tolerance and focus in particular on the concepts of tolerance mediated by regulatory $\mathrm{T}$ cells. Regulatory $\mathrm{T}$ cells are a T-cell subpopulation that acts in a dominant manner to suppress T-cell activation and expansion. The authors describe new tools to identify regulatory $\mathrm{T}$ cells, and they discuss the physiological function and the molecular characteristics of regulatory $\mathrm{T}$ cells as well as their role in a broad spectrum of disease conditions [11].

Heat shock proteins are evolutionary conserved molecules that can be up-regulated by all cells under conditions of stress. They maintain the integrity of cellular proteins and are of importance for the survival of prokaryotic and eukaryotic cells. Accumulating evidence indicates that heat shock proteins have immunoregulatory potential. Thus, a regulatory phenotype of T cells may be induced by heat shock proteins. Willem van Eden, The Netherlands, reviews immune responses induced by heat shock proteins as antigens and summarizes the potential of such responses to control inflammation [12].

The contributions to this issue of TRANSFUSION MEDICINE AND HEMOTHERAPY may stimulate a discussion process with respect to the development of new strategies to understand and to influence what we call 'compatibility' in immunohematology. The issue has become possible by the strong commitment of the authors who accepted the invitation to contribute to this issue and who were willing to share their experimental data and to let the reader participate in new and original approaches to understand immune responses. For an in-depth comprehension of the context of their work, readers are referred to the detailed up-to-date reference lists accompanying the reviews. I would like to express my sincere gratitude to all of the colleagues participating in this issue of TRANSFUSION Medicine And Hemotherapy. It has been a great pleasure to work together on this occasion.

\section{References}

1 Silverstein AM: Horror autotoxicus, autoimmunity and immune regulation: the early history. Transfus Med Hemother 2005;32:296-302.

2 Bocharov G: Understanding complex regulatory systems: integrating molecular biology and systems analysis. Transfus Med Hemother 2005;32:304-321.

3 Miller JFAP: Principles of immunological tolerance. Transfus Med Hemother 2005;32:322-331.

4 Berek C: Somatic hypermutation and B-cell receptor selection as regulators of the immune response. Transfus Med Hemother 2005;32:333-338.

5 Nielsen CH, Leslie RGQ: Regulation of B-cell activation by complement receptors and $\mathrm{Fc}$ receptors. Transfus Med Hemother 2005;32:339-347.
6 Heyman B: Antibody feedback inhibition - a biological principle of immune regulation. Transfus Med Hemother 2005;32:348-354

7 Sánchez Crespo M: Immune complex processing: a phagocytosis-based mechanism with proinflammatory potential. Transfus Med Hemother 2005;32: 355-362.

8 Scandella E, Ludewig B: Dendritic cells and autoimmunity. Transfus Med Hemother 2005;32: 363-368.

9 Ephrem A, Bayary J, Misra N, Dasgupta S, Wootla B, Van Huyen J-PD, Hassan G, Delignat S, Kaveri SV: Immunoglobulin-dependent regulation of dendritic cells in the context of autoimmune responses. Transfus Med Hemother 2005;32:369-372.
10 Iruretagoyena MI, Lezana JP, Kalergis AM: Interactions at the dendritic cell / T-cell interface define the balance between immunity and tolerance. Transfus Med Hemother 2005;32:373-383.

11 Buer J, Westendorf AM, Zeng A-P, He F, Hansen W, Probst-Kepper M: Mechanisms of central and peripheral T-cell tolerance: An update. Transfus Med Hemother 2005;32:384-399.

12 van Eden W: The immuno-regulatory T-cell response to heat shock proteins and suppression of experimental autoimmunity. Transfus Med Hemother 2005;32:400-409. 